\title{
AN EXTENSION OF THE TAYLOR SUMMABILITY TRANSFORM ${ }^{1}$
}

\author{
J. P. KING
}

1. Introduction. Two matrix methods of summability which have been the object of much recent research are the Euler matrix, $E(r)=\left(a_{n k}\right)$, and the Taylor matrix, $T(r)=\left(b_{n k}\right)$. The elements of these matrices are defined by

$$
a_{n k}= \begin{cases}\left(\begin{array}{l}
n \\
k
\end{array}\right) r^{k}(1-r)^{n-k} & (k \leqq n), \\
0 & (k>n),\end{cases}
$$

and

$$
b_{n k}= \begin{cases}0 & (k<n), \\
\left(\begin{array}{l}
k \\
n
\end{array}\right) r^{k-n}(1-r)^{n+1} & (k \geqq n),\end{cases}
$$

respectively.

R. P. Agnew [1] has studied the matrix $E\left(r_{n}\right)$ which is obtained from the $E(r)$ matrix by replacing the parameter $r$ by a parameter $r_{n}$ which is dependent on $n$. This matrix has also been studied by WuytsTorfs [4], [5]. It is natural to form the matrix $T\left(r_{n}\right)$ from the $T(r)$ matrix in a similar manner. The elements $c_{n k}$ of the $T\left(r_{n}\right)$ matrix are then defined by

$$
c_{n k}= \begin{cases}0 & (k<n), \\
\left(\begin{array}{l}
k \\
n
\end{array}\right) r_{n}^{k-n}\left(1-r_{n}\right)^{n+1} & (k \geqq n) .\end{cases}
$$

In $\$ 2$ we determine necessary and sufficient conditions on $r_{n}$ in order that $T\left(r_{n}\right)$ be regular. In $\$ 3$ we determine sufficient conditions on $r_{n}$ which insure that $T\left(r_{n}\right) \supset T(r)$, i.e., which insure that each sequence which is summable $T(r)$ is summable $T\left(r_{n}\right)$ to the same value.

2. Regularity of $T\left(r_{n}\right)$. A matrix $A=\left(a_{n k}\right)$ is regular if and only if the well-known Silverman-Toeplitz conditions:

$$
\lim _{n} a_{n k}=0 \quad(k=0,1,2, \cdots),
$$

Received by the editors August 22, 1963.

${ }^{1}$ The results of this note appear in the author's doctoral dissertation written at the University of Kentucky in 1962 under the direction of Professor V. F. Cowling. 


$$
\begin{aligned}
& \lim _{n} \sum_{k=0}^{\infty} a_{n k}=1, \\
& \sup _{n} \sum_{k=0}^{\infty}\left|a_{n k}\right|<\infty,
\end{aligned}
$$

are satisfied.

Condition (2.1) holds trivially for the $T\left(r_{n}\right)$ matrix without restriction on $r_{n}$. Condition (2.2) is satisfied if and only if $\left|r_{n}\right|<1$ $(n=0,1, \cdots)$ since

$$
\sum_{k=n}^{\infty}\left(\begin{array}{l}
k \\
n
\end{array}\right) r_{n}^{k-n}\left(1-r_{n}\right)^{n+1}=1 \quad(n=0,1, \cdots),
$$

when $\left|r_{n}\right|<1$ and the series diverges if $\left|r_{n}\right| \geqq 1$. Furthermore, if $\left|r_{n}\right|<1$,

$$
\sum_{k=n}^{\infty}\left(\begin{array}{l}
k \\
n
\end{array}\right)\left|r_{n}\right|^{k-n}\left|1-r_{n}\right|^{n+1}=\left[\frac{\left|1-r_{n}\right|}{1-\left|r_{n}\right|}\right]^{n+1},
$$

so that (2.3) will hold if and only if

$$
\left[\frac{\left|1-r_{n}\right|}{1-\left|r_{n}\right|}\right]^{n+1} \leqq K \quad(n=0,1, \cdots),
$$

where $K$ is a constant independent of $n$. That is, (2.3) will hold if and only if $\exp \left[(n+1) \log \left(\left|1-r_{n}\right| /\left(1-\left|r_{n}\right|\right)\right)\right]$ is a bounded function of $n$. We therefore have the result:

THEOREM 2.1. The $T\left(r_{n}\right)$ matrix is regular if and only if $\left|r_{n}\right|<1$ $(n=0,1, \cdots)$ and $(n+1) \log \left(\left|1-r_{n}\right| /\left(1-\left|r_{n}\right|\right)\right)$ is a bounded function of $n$.

The above theorem may be given the following geometrical characterization:

TheOREM 2.2. Let $M$ be a non-negative constant and let $k_{n}$ $=\exp (M /(n+1))(n=0,1, \cdots)$. Let $I_{n}$ denote the inner loop of the limaçon $L_{n}$ given by:

$$
p_{n}=2 \frac{k_{n}^{2} \cos \phi_{n}-k_{n}}{k_{n}^{2}-1}
$$

where $\left(p_{n}, \phi_{n}\right)$ are polar coordinates at $z=1$ with $\phi_{n}$ measured clockwise from the line segment joining $z=0$ and $z=1$. Then $T\left(r_{n}\right)$ is regular if and only if $\left|r_{n}\right|<1$ and $r_{n} \in I_{n}(n=0,1, \cdots)$. 
Proof. Condition (2.4) holds if and only if

$$
\frac{\left|1-r_{n}\right|}{1-\left|r_{n}\right|} \leqq e^{M /(n+1)} \quad(n=0,1, \cdots),
$$

where $M$ is a non-negative constant. For fixed $n$ this last inequality is satisfied in a region bounded by the curve $L_{n}$ given by

$$
\left|1-r_{n}\right|=k_{n}\left(1-\left|r_{n}\right|\right) \text {. }
$$

Introducing polar coordinates $\left(p_{n}, \phi_{n}\right)$ as described in the statement of Theorem 2.2, we have

$$
1-r_{n}=p_{n}\left(\cos \phi_{n}+i \sin \phi_{n}\right) \text {. }
$$

Equation (2.6) becomes

$$
p_{n}=k_{n}\left[1-\left|1-p_{n}\left(\cos \phi_{n}+i \sin \phi_{n}\right)\right|\right] \text {. }
$$

Hence

$$
\left(p_{n}-k_{n}\right)^{2}=k_{n}^{2}\left(1+2 k_{n} \cos \phi_{n}+p_{n}^{2}\right),
$$

so that

$$
p_{n}\left(k_{n}^{2}-1\right)-2 k_{n}^{2} \cos \phi_{n}+2 k_{n}=0,
$$

which reduces to (2.5).

For fixed $n$ this is the equation of a limaçon with two branches through the point $z=1$. Since $\left|r_{n}\right|<1$, in order that $T\left(r_{n}\right)$ be regular, it is clear that $r_{n}$ must lie in the inner loop $I_{n}$ of $L_{n}$. This proves the theorem.

It is useful to note that the sequence of regions $I_{n}$ approaches the interval $[0,1)$ as $n \rightarrow \infty$. To see this we notice that $p_{n}=2 k_{n} /\left(k_{n}+1\right)$ when $\phi_{n}=0$, and that this quantity tends to 1 as $n \rightarrow \infty$. It is an exercise in elementary calculus to prove that the maximum height of the boundary of $I_{n}$ above the $x$-axis tends to 0 as $n \rightarrow \infty$.

We may note also that, in particular, $T\left(r_{n}\right)$ is regular if $0 \leqq r_{n}<1$ $(n=0,1, \cdots)$. Furthermore, if $r_{n}=r$, a constant $(n=0,1, \cdots)$, then $T\left(r_{n}\right)=T(r)$ is regular if and only if $0 \leqq r<1$. This is the known necessary and sufficient condition that $T(r)$ be regular [2].

3. The relation $T\left(r_{n}\right) \supset T(r)$. Let $\left\{s_{i}\right\}$ be a sequence which is $T(r)$-summable to $s$, where $r \neq 1$. Let $\left\{u_{k}\right\}$ denote the $T(r)$-transform of the sequence $\left\{s_{i}\right\}$ and let $\left\{t_{k}\right\}$ denote the $T\left(r_{n}\right)$-transform of the same sequence. It is known [2] that if $r \neq 1$, the $T(r)$ matrix has the inverse $T(p)$ where $p=r /(r-1)$. We therefore have 


$$
t_{n}=\sum_{m=n}^{\infty}\left(\begin{array}{l}
m \\
n
\end{array}\right) r_{n}^{m-n}\left(1-r_{n}\right)^{n+1} \sum_{k=m}^{\infty}(1-p)^{m+1}\left(\begin{array}{l}
k \\
m
\end{array}\right) p^{k-m} u_{k} .
$$

If we formally interchange the order of summation we have

$$
t_{n}=\sum_{k=1}^{\infty}\left(1-r_{n}\right)^{n+1} u_{k} \sum_{m=n}^{k}\left(\begin{array}{l}
m \\
n
\end{array}\right)\left(\begin{array}{l}
k \\
m
\end{array}\right) r_{n}^{m-n} p^{k-m}(1-p)^{m+1} \text {. }
$$

The interchange of summation will be justified provided the series

$$
R=\sum_{k=n}^{\infty}\left|1-r_{n}\right|^{n+1}\left|u_{k}\right| \sum_{m=n}^{k}\left(\begin{array}{l}
m \\
n
\end{array}\right)\left(\begin{array}{l}
k \\
m
\end{array}\right)\left|r_{n}\right|^{m-n}|p|^{k-m}|1-p|^{m+1}
$$

converges. Since the sequence $\left\{u_{k}\right\}$ converges there exists a constant $M$ such that $\left|u_{k}\right| \leqq M(k=0,1, \cdots)$. Therefore

$$
\begin{aligned}
R & \leqq M \sum_{k=n}^{\infty}\left|1-r_{n}\right|^{n+1}\left(\begin{array}{l}
k \\
n
\end{array}\right) \sum_{m=n}^{k}\left(\begin{array}{c}
k-n \\
m-n
\end{array}\right)\left|r_{n}\right|^{m-n}|p|^{k-m}|1-p|^{m+1} \\
& \leqq M \sum_{k=n}^{\infty}\left|1-r_{n}\right|^{n+1}\left(\begin{array}{l}
k \\
n
\end{array}\right)|1-p|^{n+1}\left[\left|r_{n}\right||1-p|+|p|\right]^{k-n} \\
& \leqq M \frac{\left|1-r_{n}\right|^{n+1}|1-p|^{n+1}}{\left[1-\left(\left|r_{n}\right||1-p|+|p|\right)\right]^{n+1}},
\end{aligned}
$$

provided $\left|r_{n}\right||1-p|+|p|<1$. That is, provided

$$
\left|r_{n}\right|+|r|<|1-r| \text {. }
$$

Consequently, if (3.1) holds we have

$$
\begin{aligned}
t_{n} & =\sum_{k=n}^{\infty}\left(\begin{array}{l}
k \\
n
\end{array}\right)\left(1-r_{n}\right)^{n+1} u_{k} \sum_{v=0}^{k-n}\left(\begin{array}{c}
k-n \\
v
\end{array}\right) r_{n}^{0} p^{k-n-v}(1-p)^{n+v+1} \\
& =\sum_{k=n}^{\infty}\left(\begin{array}{l}
k \\
n
\end{array}\right)\left(1-r_{n}\right)^{n+1}(1-p)^{n+1}\left(r_{n}-r_{n} p+p\right)^{k-n} u_{k} \\
& =\sum_{k=n}^{\infty}\left(\begin{array}{l}
k \\
n
\end{array}\right)\left[1-\left(r_{n}+p-r_{n} p\right)\right]^{n+1}\left(r_{n}-r_{n} p+p\right)^{k-n} u_{k} \\
& =\sum_{k=n}^{\infty}\left(\begin{array}{l}
k \\
n
\end{array}\right)\left(1-x_{n}\right)^{n+1} x_{n}^{k-n} u_{k},
\end{aligned}
$$

where $x_{n}=r_{n}+p-r_{n} p=\left(r-r_{n}\right) /(r-1)$. It is clear that $\left\{t_{n}\right\}$ will converge to $s$, and hence $T\left(r_{n}\right) \supset T(r)$, if the matrix $T\left(x_{n}\right)$ is regular. This will be true if the conditions of Theorem 2.1 are satisfied for $T\left(x_{n}\right)$. We have therefore the result: 
THEOREM 3.1. If $r \neq 1$ is a complex constant such that (3.1) holds then $T\left(r_{n}\right) \supset T(r)$ if

$$
\left|\frac{r_{n}-r}{1-r}\right|<1 \quad(n=0,1, \cdots),
$$

and

$$
(n+1) \log \frac{\left|1-r_{n}\right|}{|1-r|-\left|r_{n}-r\right|}<M \quad(n=0,1, \cdots)
$$

where $M$ is a constant independent of $n$.

We may note that if $r_{n}=r_{2}=$ constant $(n=0,1, \cdots)$, then (3.2) and (3.3) hold if and only if

$$
\left|1-r_{2}\right|=|1-r|-\left|r_{2}-r\right| \text {. }
$$

This gives:

COROLLARY 3.2. If $r \neq 1,\left|r_{2}\right|+|r|<|1-r|$, and if (3.4) holds, then $T\left(r_{2}\right) \supset T(r)$.

This last result was first proved by Laush [3], who also proved that the conditions of Corollary 3.2 are necessary if $0<|r|<\left|r_{2}\right|<1$.

\section{REFERENCES}

1. R. P. Agnew, Euler transformations, Amer. J. Math. 66 (1944), 318-338.

2. V. F. Cowling, Summability and analytic continuation, Proc. Amer. Math. Soc. 1 (1950), 536-542.

3. G. Laush, Relations among the Weierstrass methods of summability, Doctoral Dissertation, Cornell University, Ithaca, N. Y., 1949.

4. M. Wuyts-Torfs, On a regular generalization of the Euler summation method, Simon Stevin 32 (1958), 170-175. (Dutch)

5. - On a generalization of the Euler limit method, Simon Stevin 33 (1959), 27-33. (Dutch)

LEHIGH UNIVERSITY 